Neurosurg Focus 24 (6):E6, 2008

\title{
Brain abscess in children
}

\author{
Jason P. Sheehan, M.D., Ph.D., ${ }^{1}$ John A. Jane JR., M.D., ${ }^{1,2}$ Dibyendu K. RaY, M.D., ${ }^{1}$ AND \\ Howard P. Goodkin, M.D., PH.D. ${ }^{2,3}$
}

Departments of ${ }^{I}$ Neurosurgery, ${ }^{2}$ Pediatrics, and ${ }^{3}$ Neurology, University of Virginia Health System, Charlottesville, Virginia

\begin{abstract}
$\checkmark$ Although it is uncommon, pediatric brain abscess remains a serious, life-threatening neurological problem. Those with congenital heart disease, an ongoing infection, or an immunocompromised state are particularly at risk. The symptoms on presentation may include those associated with a space-occupying lesion in the brain, and neuroimaging has made the diagnosis of brain abscess more reliable. Prompt diagnosis and treatment are required to lessen neurological morbidity and the risk of death. Treatment includes medical management with appropriate and specific antimicrobials. Although the effectiveness of medical management has improved and some children may be treated with antimicrobial therapy alone, surgical evaluation remains an important component of the treatment algorithm for most pediatric patients. (DOI: $10.3171 /$ FOC/2008/24/6/E6)
\end{abstract}

KEY WORDS • brain abscess • children • infection

A BRAIN abscess is an intraparenchymal infection that commences as a localized area of cerebritis evolving through various stages into a collection of encapsulated purulent material. ${ }^{3}$ The infection results from the seeding of the parenchyma as the result of hematogenous spread from remote sources, direct invasion from contiguous infection of nonneural tissues, or from implantation of pathogens following penetrating wounds or surgery. Once formed, the abscess can result in permanent neurological disability by direct destruction, infarction, or compression. Although advances in head imaging resulted in an initial decline in the mortality rate, children with brain abscesses do remain at risk for death. , $^{1,910,23,25}$ Early diagnosis combined with the prompt initiation of empirical broadspectrum antimicrobial therapy and neurosurgical intervention are important components in the care and treatment of children with brain abscesses.

Brain abscesses in children are rare. ${ }^{12,15}$ At the University of Virginia Children's Hospital for the years 2000-2007 (inclusive), an average of 1.5 children per year was admitted to the inpatient pediatric service with a primary diagnosis of brain abscess.

The most common risk factors that predispose a child to the formation of a brain abscess include CHD, sinus and otogenic infections, poor dental hygiene and complications from dental procedures, infancy, immunosuppression, neurosurgical procedures such as implantation of ventriculoperitoneal shunts, penetrating skull injury and comminuted

Abbreviations used in this paper: $\mathrm{CHD}=$ congenital heart disease; $\mathrm{CSF}=$ cerebrospinal fluid; $\mathrm{MR}=$ magnetic resonance. fracture of the skull, congenital lesions of the head and neck (such as dermal sinuses), and as a rare complication of meningitis. In addition, there have been case reports of brain abscess in children following aspiration of foreign bodies, ${ }^{21}$ esophageal endoscopy, ${ }^{18}$ ocular trauma, ${ }^{2}$ and placement of dental braces. ${ }^{26}$ In a significant proportion of children, however, a predisposing factor is not identified.

Goodkin and colleagues ${ }^{10}$ characterized the natural history of brain abscess at Children's Hospital Boston between the years 1981 and 2000. During that time period, CHD and sinus/otitic infections were the most common predisposing factors. When compared with a similar study performed at the same institution for the years 1945-1980, ${ }^{9}$ there were several interesting differences. Although CHD was the most common predisposing factor in both studies, there was a reduction in the number of brain abscesses in the setting of sinus and otitic infections (also see Carpenter et al.5). These authors hypothesized that this decrease resulted from the increased use and efficacy of antibiotics for the treatment of these infections during the later time period. ${ }^{19}$ Second, there was an increase in the number of infections occurring in immunocompromised children. These abscesses were usually a severe consequence of disseminated fungal disease, and 6 of the 9 patients identified as having intracerebral fungal abscesses died. Last, the number of neonates identified as having brain abscesses increased.

Abscesses may be single or multiple, and the lesion's cerebral localization is often related to the underlying, predisposing factor. Abscesses that are the result of hematogenous spread from distant sources such as the heart (endo- 
carditis) or lungs may have a distribution that reflects the cerebral arterial supply, most commonly that of the middle cerebral artery. Hematogenous spread can also occur by way of the veins that drain into the cavernous sinus, resulting in frontal lobe abscesses that are correlated with infections of the facial tissues or ethmoidal sinuses. Oral microorganisms may also spread to the frontal lobe in this manner or enter the cranial vault through direct invasion. Infections of the middle ear spread by direct invasion that may result in abscesses in the temporal lobe or cerebellum. The location tends to vary with a child's age; cerebellar abscesses are more common in younger children and temporal lobe abscesses in older children. Abscesses of the brainstem are rare and can be observed in cases with either otogenic or hematogenous sources.

The most commonly identified causative microorganisms include the streptococci (aerobic and anaerobic) and staphylococci (S. aureus and other staphylococcal species). Nevertheless, a wide range of bacteria, including other gram-positive organisms (for example, peptostreptococci), gram-negative organisms (for example, Haemophilus species), fungi (Aspergillus species), and parasites have been recovered from brain abscesses. In the Children's Hospital Boston study, ${ }^{10} \mathrm{~S}$. milleri represented the most common organism in children beyond the neonatal period, and $\mathrm{Ci}$ trobacter species were identified as the causative microorganism in 3 of the 5 infections in children $<1$ month old. Because many abscesses will contain mixed flora (39\% of cases in the Children's Hospital Boston study), ${ }^{10}$ empirical broad-spectrum antibiotic therapy is necessary prior to isolation of the causative agent or agents (see below).

\section{Clinical Features}

It is imperative to note that the classic triad of fever, headache, and neurological deficit may be incomplete at the time of presentation. ${ }^{1,9,10}$ Brain abscess should be a strong consideration in the child who presents with the new onset of acute headaches or first-time seizure, especially when focal neurological signs are present on examination. In the neonate, a brain abscess is a potential cause of irritability, a bulging fontanelle, and a rapid increase in head circumference.

On presentation, a child's mental status can vary from mild confusion to lethargy to stupor or coma. In the Children's Hospital Boston studies, ${ }^{9,10}$ presentation in coma was less common during the years 1981-2000 compared with 1945-1980, probably due to advancements in neuroimaging techniques resulting in earlier diagnosis. Focal neurological signs are not always present. However, if symptomatic, neurological signs will vary with abscess location. Frontal lobe abscesses may be silent until quite large, and they may result in personality change, frontal release signs, and hemiparesis. With abscesses in the temporal lobe, dysphasias may be present when the abscess is located in the dominant hemisphere, and visual field deficits ranging from contralateral upper-quadrant field cuts to complete homonymous hemianopia may be observed. With parietal lobe abscesses, there may be visual field cuts ranging from an inferior quadrantanopia to homonymous hemianopia; dysphasias when the abscess is located in the dominant hemisphere; or dyspraxia and spatial neglect when the ab- scess involves the nondominant hemisphere. Cerebellar abscesses can produce appendicular and gait ataxias and eye movement abnormalities. Brainstem abscesses are likely to result in a combination of cranial nerve palsies and deficits of ascending and descending pathways.

As an abscess grows, intracranial pressure will increase and there is the potential for herniation. Therefore, the presence of papilledema noted on physical examination requires prompt radiological evaluation and the initiation of measures to reduce intracranial pressure, such as the administration of corticosteroids and consideration of immediate neurosurgical intervention. In addition, abscess growth can result in rupture of the abscess into the ventricular system. This life-threatening event will result in an acute decompensation and symptoms of a purulent meningitis.

\section{Diagnosis and Management}

Modern-day imaging techniques such as cranial computed tomography scanning and MR imaging of the head allow the prompt confirmation of the clinical diagnosis and for the determination of abscess location and number (Fig. 1). Today, MR imaging is the study of choice. ${ }^{11}$ Diffusionweighted MR imaging and MR spectroscopy can be helpful in cases in which it is difficult to differentiate a brain abscess from a tumor, ${ }^{6,8}$ (Fig. 1C and 1D) and potentially to differentiate between fungal and bacterial sources. ${ }^{20}$ If an MR image cannot be obtained, a computed tomography scan with intravenous contrast should be performed; or, in the neonate, bedside cranial ultrasonography is another alternative. ${ }^{24}$

Once the diagnosis is confirmed, additional studies with the goal of identifying the predisposing factors and the source of infection will be guided by the patient's history and physical examination. Examination of the teeth is an important component of the physical examination of a child with an intracerebral abscess. If endocarditis is suspected, blood cultures and an echocardiogram should be obtained. If there has been direct invasion from the sinuses or middle ear, the imaging of the head should include these regions.

Blood cultures are rarely positive and obtaining CSF by lumbar puncture in the presence of a brain abscess can be life threatening. In those cases in which CSF has been obtained, there may be a mild mononuclear pleocytosis, slight elevation of protein, and a normal concentration of glucose; however, the culture tends to be sterile unless the abscess has ruptured into the ventricular system.

Although cerebritis, small solitary abscesses $(<2 \mathrm{~cm}$ in diameter), or those in which the causative agent has been identified can be treated with antimicrobials alone, $, 13,17,22$ strong consideration should be given to surgical drainage followed by the prompt initiation of empirical broad-spectrum empiric antimicrobial therapy (Table 1). The regimen can be refined once the offending organism or organisms and susceptibilities have been identified in the neurologically stable child in whom the abscess or abscesses are accessible. The surgical management of brain abscesses in children is similar to that of these lesions in adults. Frequently, especially in children in whom the abscess is the result of direct invasion of a contiguous infection involving the sinuses or middle ear, a multidisciplinary approach that 

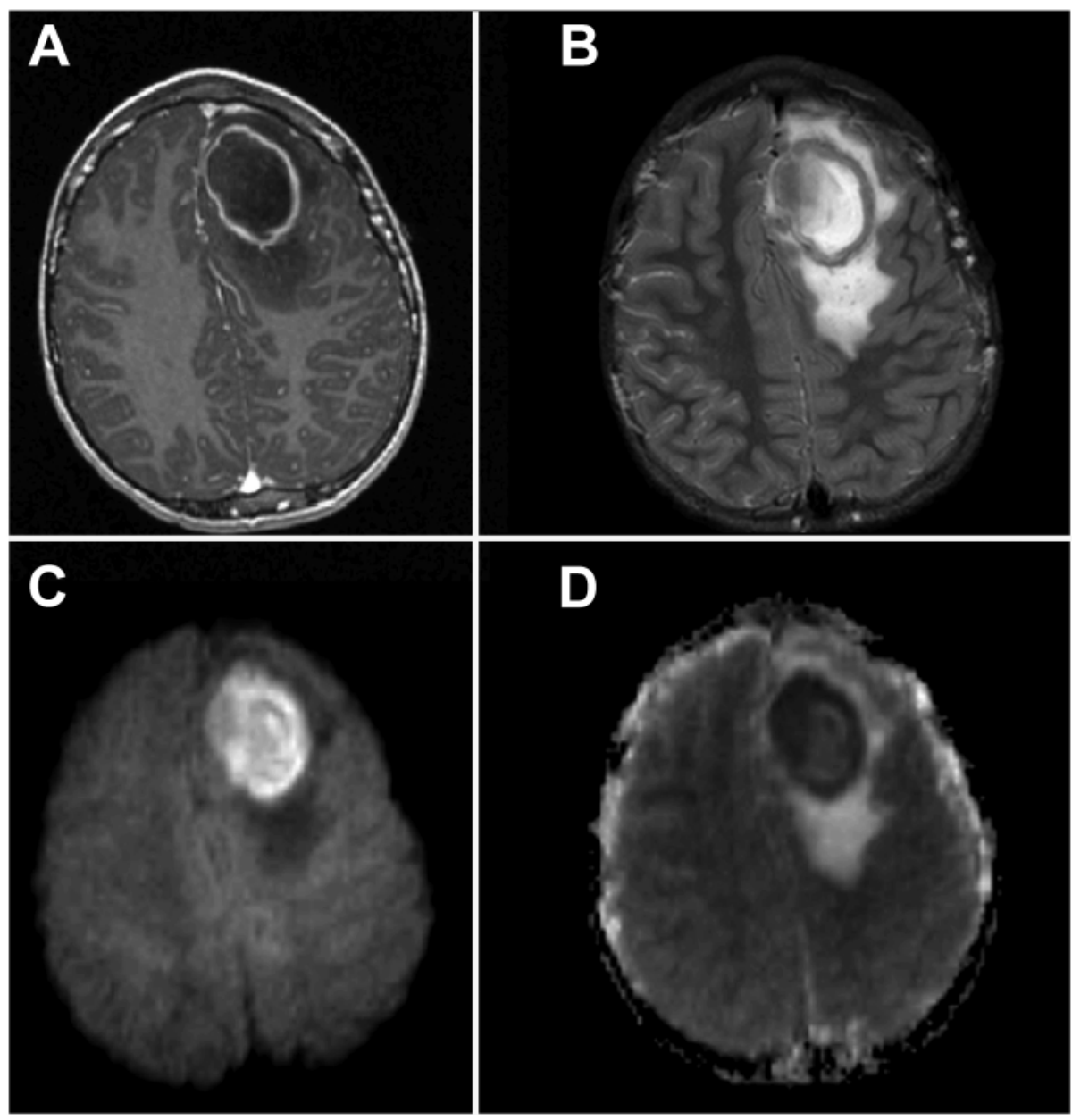

FIG. 1. Axial MR images of a left-sided frontal pyogenic abscess in a 7-year-old boy. Axial contrast-enhanced T1weighted image (A) demonstrating a well-defined, relatively uniform, thin enhancing rim. On an axial T2-weighted image (B), the rim is hypointense due to collagen, hemorrhage, or paramagnetic free radicals; this is an MR feature characteristic of abscess capsules. Hyperintense vasogenic edema surrounds the abscess. Axial diffusion-weighted image (C) and apparent diffusion coefficient map (D) show that the central pus is hyperintense and hypointense, respectively, findings that help distinguish this abscess from a necrotic neoplasm.

includes both the neurosurgeon and the otolaryngologist is required. Surgical drainage can often be performed using frameless stereotactic bur hole aspiration. Preoperative stereotactic imaging allows for computation of the abscess volume and provides an estimate of how much material can reasonably be expected to be withdrawn. Use of intraoperative ultrasonography can help further to delineate pockets of abscess. In our experience as well as in the Children's Hospital Boston study, ${ }^{10}$ the majority of children only require a single aspiration. However, children with new neurological signs or continued abscess growth despite antimicrobial therapy that is deemed appropriate based on the causative microorganism require additional surgical procedures. In children in whom the abscess remains enlarged despite repeated needle aspiration and medical management, strong consideration should be given to the performance of a craniotomy to allow more adequate evacuation of the purulent material and debridement of the surrounding parenchyma. For those abscesses associated with a foreign body such as a shunt, brain-stimulating electrode, or other device, the foreign body should be removed at the time of the abscess evacuation and the abscess capsule should be microsurgically removed at the time of the craniotomy. Surgical complications associated with aspiration and resection include hemorrhage, CSF leakage, seizure, and stroke. In addition, while aspirating the abscess, great care should be taken to avoid a needle trajectory through the ventricular system.

Initial antimicrobial therapy is administered with broadspectrum agents such as a third-generation cephalosporin and metronidazole. Therapy can be narrowed if a specific organism or organisms are identified. It is difficult to isolate anaerobic bacteria even if they are part of the microbiological composition of the cerebral abscess, so anaerobic coverage is often maintained if only one organism is identified within the culture. There are no prospective studies in children to guide the duration of antimicrobial therapy; however, in immunocompetent hosts we tend to favor intravenous antimicrobial courses at least 4-6 weeks in duration (and longer in immunosuppressed patients or those with tubercular brain abscess), with long-term follow-up head imaging to ensure that the abscess or abscesses have 
TABLE 1

Common causative organisms of brain abscess in children, stratified by predisposing factors

\begin{tabular}{ll}
\hline \hline \multicolumn{1}{c}{ Predisposing Factor } & \multicolumn{1}{c}{ Causative Organism(s) } \\
\hline neonate & Proteus spp \\
& Citrobacter spp \\
& Enterobacter spp \\
immunocompromised host & Nocardia spp \\
& fungi \\
& Mycobacterium tuberculosis \\
CHD & S. viridans \\
& microaerophilic streptococci \\
& Haemophilus spp \\
infection & streptococci (aerobic \& anaerobic) \\
middle ear & Enterobacteriaceae \\
& Pseudomonas spp \\
sinus & streptococci (aerobic \& anaerobic) \\
& S. aureus \\
& Enterobacteriaceae \\
oral cavity & mixed anaerobic flora \\
& streptococci (aerobic \& anaerobic) \\
& S. aureus \\
posttraumatic & Enterobacteriaceae \\
& S. aureus \\
& streptococci spp \\
& Enterobacteriaceae \\
\hline &
\end{tabular}

resolved. Common pathogens and potential empirical antimicrobial regimens based on these suspected organisms are outlined in Tables 1 and 2.

As is the case for antibiotics, there are no studies to guide the prophylactic use of antiepileptic therapy in children with brain abscesses. Although some practitioners tend to favor short-term prophylactic use of these drugs in all children with a brain abscess involving cortical structures, others will start antiepileptic therapy only in patients whose presentation included a seizure. If anticonvulsants are started, pharmacological interactions between antibiotics and anticonvulsants must be considered in calculating dosages.

\section{Outcome}

A cerebral abscess is a destructive lesion, and therefore it is not surprising that many of the children affected will have neurological sequelae that include epilepsy, new motor deficits, persistent visual field cuts, learning disorders, and hydrocephalus requiring the placement of a ventriculoperitoneal shunt. $7,9,10,12,14,16$

Improved imaging techniques combined with improved culturing techniques and advances in surgical and medical management of brain abscesses have contributed to the reduction in the mortality rate, which has been seen over the last century. ${ }^{9,25}$ However, this condition remains life threatening, especially in children who are immunocompromised, who are $<1$ year old, who present with significant surgery-related neurological deficits, in whom the initial diagnosis was delayed, the abscess has ruptured into the ventricle, or surgical management was complicated by hemorrhage.

\section{Conclusions}

Pediatric brain abscesses are a serious and potentially
TABLE 2

Potential empirical therapy, stratified by predisposing factor*

\begin{tabular}{ll}
\hline \hline Predisposing Factor & \multicolumn{1}{c}{ Potential Empirical Therapy } \\
\hline $\begin{array}{l}\text { neonate } \\
\text { immunocompromised } \\
\text { host }\end{array}$ & $\begin{array}{l}\text { cefotaxime plus ampicillin } \\
\text { vancomycin plus ceftazidine plus metronidazole } \\
\text { consider amphotericin \& antituberculosis therapy } \\
\text { TMP-SMZ for Nocardia spp } \\
\text { CHD }\end{array}$ \\
$\begin{array}{l}\text { ceftriaxone plus metronidazole } \\
\text { consider vancomycin } \\
\text { middle ear } \\
\text { sinus }\end{array}$ & $\begin{array}{l}\text { ceftriaxone plus metronidazole } \\
\text { ceftriaxone plus metronidazole } \\
\text { consider therapy for MRSA } \\
\text { penicillin plus metronidazole or ampicillin- } \\
\text { sulbactam }\end{array}$ \\
posttraumatic & vancomycin plus 3rd-generation cephalosporin \\
\hline
\end{tabular}

* MRSA = methicillin-resistant $S$. aureus; TMP-SMZ = trimethoprimsulfamethoxazole.

life-threatening intracranial entity for which prompt diagnosis and treatment are required. Serial neuroimaging must be performed to document an improvement in and eventually a resolution of the infection. Surgical treatment with either needle aspiration or excision remains an important part of the treatment algorithm for pediatric patients afflicted with brain abscesses.

\section{Disclosure}

Dr. Goodkin receives support from the National Institutes of Health (Grant No. NS-048413). The authors have no other financial disclosures.

\section{Acknowledgments}

We thank J. Owen Hendley and Linda Waggoner-Fountain for their thoughtful comments and assistance in the completion of this manuscript. We also thank Dr. Julie Matsumoto of the Department of Radiology at the University of Virginia Health System for the images in Fig. 1.

\section{References}

1. Auvichayapat N, Auvichayapat P, Aungwarawong S: Brain abscess in infants and children: a retrospective study of 107 patients in northeast Thailand. J Med Assoc Thai 90:1601-1607, 2007

2. Bank DE, Carolan PL: Cerebral abscess formation following ocular trauma: a hazard associated with common wooden toys. Pediatr Emerg Care 9:285-288, 1993

3. Britt RH, Enzmann DR, Yeager AS: Neuropathological and computerized tomographic findings in experimental brain abscess. J Neurosurg 55:590-603, 1981

4. Brook I: Brain abscess in children: microbiology and management. J Child Neurol 10:283-288, 1995

5. Carpenter J, Stapleton S, Holliman R: Retrospective analysis of 49 cases of brain abscess and review of the literature. Eur J Clin Microbiol Infect Dis 26:1-11, 2007

6. Castillo M: Imaging brain abscesses with diffusion-weighted and other sequences. AJNR Am J Neuroradiol 20:1193-1194, 1999

7. Ciurea AV, Stoica F, Vasilescu G, Nuteanu L: Neurosurgical management of brain abscesses in children. Childs Nerv Syst 15: 309-317, 1999

8. Desprechins B, Stadnik T, Koerts G, Shabana W, Breucq C, Osteaux M: Use of diffusion-weighted MR imaging in differential diagnosis between intracerebral necrotic tumors and cerebral abscesses. AJNR Am J Neuroradiol 20:1252-1257, 1999 


\section{Pediatric brain abscess}

9. Fischer EG, McLennan JE, Suzuki Y: Cerebral abscess in children. Am J Dis Child 135:746-749, 1981

10. Goodkin HP, Harper MB, Pomeroy SL: Intracerebral abscess in children: historical trends at Children's Hospital Boston. Pediatrics 113:1765-1770, 2004

11. Haimes AB, Zimmerman RD, Morgello S, Weingarten K, Becker RD, Jennis R, et al: MR imaging of brain abscesses. AJR Am J Radiol 152:1073-1085, 1989

12. Hegde AS, Venkataramana NK, Das BS: Brain abscess in children. Childs Nerv Syst 2:90-92, 1986

13. Heineman HS, Braude AI, Osterholm JL: Intracranial suppurative disease. Early presumptive diagnosis and successful treatment without surgery. JAMA 218:1542-1547, 1971

14. Idriss ZH, Gutman LT, Kronfol NM: Brain abscesses in infants and children: current status of clinical findings, management and prognosis. Clin Pediatr (Phila) 17:738-740, 745-746, 1978

15. Jadavji T, Humphreys RP, Prober CG: Brain abscesses in infants and children. Pediatr Infect Dis 4:394-398, 1985

16. Legg NJ, Gupta PC, Scott DF: Epilepsy following cerebral abscess. A clinical and EEG study of 70 patients. Brain 96: 259-268, 1973

17. Liston TE, Tomasovic JJ, Stevens EA: Early diagnosis and management of cerebritis in a child. Pediatrics 65:484-486, 1980

18. Louie JP, Osterhoudt KC, Christian CW: Brain abscess following delayed endoscopic removal of an initially asymptomatic esophageal coin. Pediatr Emerg Care 16:102-105, 2000

19. McCaig LF, Besser RE, Hughes JM: Trends in antimicrobial prescribing rates for children and adolescents. JAMA 287: 3096-3102, 2002
20. Mueller-Mang C, Castillo M, Mang TG, Cartes-Zumelzu F, Weber M, Thurnher MM: Fungal versus bacterial brain abscesses: is diffusion-weighted MR imaging a useful tool in the differential diagnosis? Neuroradiology 49:651-657, 2007

21. Roberts J, Bartlett AH, Giannoni CM, Valdez TA: Airway foreign bodies and brain abscesses: Report of two cases and review of the literature. Int J Pediatr Otorhinolaryngol 72:265-269, 2008

22. Rosenblum ML, Hoff JT, Norman D, Edwards MS, Berg BO: Nonoperative treatment of brain abscesses in selected high-risk patients. J Neurosurg 52:217-225, 1980

23. Sennaroglu L, Sozeri B: Otogenic brain abscess: review of 41 cases. Otolaryngol Head Neck Surg 123:751-755, 2000

24. Sidaras D, Mallucci C, Pilling D, Yoxall WC: Neonatal brain abscess_-potential pitfalls of CT scanning. Childs Nerv Syst 19:57-59, 2003

25. Tekkök IH, Erbengi A: Management of brain abscess in children: review of 130 cases over a period of 21 years. Childs Nerv Syst 8:411-416, 1992

26. Wolf J, Curtis N: Brain abscess secondary to dental braces. Pediatr Infect Dis J 27:84-85, 2008

Manuscript submitted February 16, 2008.

Accepted Marach 3, 2008.

Address correspondence to: Howard P. Goodkin, M.D., Ph.D., PO Box 800394, Charlottesville, Virginia 22908. email: hpg9v @ virginia.edu. 